\title{
SOME LOWER BOUNDS ON THE NUMBER OF RESONANCES IN EUCLIDEAN SCATTERING
}

\section{T. Christiansen}

The purpose of this note is to give some new lower bounds on the number of resonances, or scattering poles, for non-trivial, real-valued, smooth, compactly supported potentials in dimension $n \geq 3$, odd. Let $N(r)$ be the number of resonances, counted with multiplicity, with norm less than $r$. We prove that

$$
\lim \sup _{r \rightarrow \infty} \frac{N(r)}{r(\log r)^{-p}}=\infty
$$

for any $p>1$; this is the first quantitative lower bound to hold in this generality. We give some similar results for scattering by non-trapping metric perturbations and for scattering by certain obstacles with fractal boundaries. The non-zero resonances are defined, equivalently, as the poles of the meromorphic continuation of the resolvent or of the scattering matrix ([6]). For the situations we consider, except, possibly, for a finite number of points, the poles of the meromorphic continuation of the resolvent correspond, with multiplicity, to the poles of the determinant of the scattering matrix $([5,22])$.

Rather little is known about the poles for general potentials. Let $V \in$ $L_{\text {comp }}^{\infty}\left(\mathbb{R}^{n}\right)$ be real-valued, $n \geq 3$ odd, and let $R(\lambda)=\left(\Delta+V-\lambda^{2}\right)^{-1}$ be the resolvent of $\Delta+V$. We take the convention that, aside from a finite number of $\lambda, R(\lambda)$ is a bounded operator on $L^{2}\left(\mathbb{R}^{n}\right)$ when $\operatorname{Im} \lambda<0$. It has a meromorphic continuation to $\mathbb{C}$ as an operator from $L_{\text {comp }}^{2}\left(\mathbb{R}^{n}\right)$ to $L_{\text {loc }}^{2}\left(\mathbb{R}^{n}\right)$. Let

$N(r)=\#\left\{\lambda_{j}: \lambda_{j}\right.$ is a pole of $R(\lambda)$, listed with multiplicity, and $\left.\left|\lambda_{j}\right|<r\right\}$.

In [21], for odd $n \geq 3$, Zworski showed that for $V \in L_{\text {comp }}^{\infty}\left(\mathbb{R}^{n}\right), N(r)=\mathcal{O}\left(r^{n}\right)$, and this bound is optimal in that there are potentials for which $N(r) \geq c r^{n}$, $c>0$; in [20] he obtained asymptotics for $N(r)$ when $n=1$. Only relatively recently has it been shown that if $V$ is a smooth, super-exponentially decaying potential, $V \not \equiv 0$, then there are infinitely many poles. This was done for $n=3$ ([12]), for a combination of a potential and metric perturbation when $n=3$ ([16]), and then for potential scattering in all odd dimensions ([17]); see [17] for a brief history of the problem.

Let $S(\lambda)$ be the scattering matrix, $s(\lambda)=\operatorname{det} S(\lambda)$, and, for $\lambda$ real, $\sigma(\lambda)=$ $(2 \pi i)^{-1} \int_{0}^{\lambda} s^{-1}(\tau) s^{\prime}(\tau) d \tau+s_{0 \pm}$ be the scattering phase, where $s_{0 \pm}=1$ if 0 is a resonance and is 0 otherwise. We use knowledge of $s(\lambda)$ and $\sigma(\lambda)$ near 0 and infinity, both of which are rather well understood in the potential case, and a

Received December 9, 1998. Revised February 11, 1999. 
representation of $s(\lambda)$ from [22] to obtain some information about $N(r)$. Our methods are in the spirit of what has been done for surfaces with cusps (e.g. $[13,14])$. However, since the results of $[13,14]$ make extensive use of the fact that most of the resonances lie near the real axis, such precise results are not possible here.

From [22] we know that $s(\lambda)= \pm e^{i g(\lambda)} P(-\lambda) / P(\lambda)$, where

$$
P(\lambda)=\prod_{\lambda_{j} \text { pole }, \lambda_{j} \neq 0} E\left(\lambda / \lambda_{j}, n\right), \quad E(z, p)=(1-z) \exp \left(z+\frac{z^{2}}{2}+\cdots+\frac{z^{p}}{p}\right),
$$

$\left\{\lambda_{j}\right\}$ are all the non-zero poles of the resolvent, including those corresponding to eigenvalues, and $g$ is a polynomial of order at most $n$. We take the positive sign when 0 is not a resonance and the negative sign otherwise ([8]). Moreover, using the fact that for real $\lambda,|s(\lambda)|=1, \sigma^{\prime}(\lambda)$ is even, and $\sigma(\lambda)$ is real, we obtain that $g(\lambda)$ is real when $\lambda$ is real, odd, and has $g(0)=0$.

In the spirit of this note, we give a different proof of the following lemma (see $[17,15]$, and references). Effectively, we are using the behaviour of the scattering phase near 0 rather than the asymptotics of the heat kernel, as was noted as a possibility by Müller (See [22, Section 2], and also [23, Section 3].). Note that it was observed by Melrose ([12]) that the existence of at least one pole is enough to show that there are infinitely many.

Lemma. The resolvent of $\Delta+V$, where $V \in C_{c}^{\infty}\left(\mathbb{R}^{n}\right)$ is real-valued, $n \geq 3$ odd, has at least one nonzero pole if $V \not \equiv 0$.

Proof. Suppose there are no non-zero poles. Then $s(\lambda)= \pm e^{i g(\lambda)}$ and $\sigma(\lambda)=$ $(2 \pi)^{-1} g(\lambda)+s_{0 \pm}$. However, from results on the behaviour of the scattering matrix near $0([7,8])$, under these assumptions $g(\lambda)=\alpha_{n-2} \lambda^{n-2}+\alpha_{n} \lambda^{n}$ where $\alpha_{n-2}, \alpha_{n}$ are constants. Since the coefficient of $\lambda^{n-4}$ in the expansion at infinity of the scattering phase is a nonzero multiple of $\int V^{2}(x) d x([1,3,4])$, it follows that $V \equiv 0$.

We shall use the following notation: For $f, g \geq 0$, we say that $f(r)=\Omega(g(r))$ as $r \rightarrow \infty$ if for any $C>0, R>0$, there is an $r_{1}>R$ with $f\left(r_{1}\right)>C g\left(r_{1}\right)$.

We note that if $\sum_{m=1}^{\infty}\left|a_{m}\right|^{-p-1}<\infty$, then $\prod_{1}^{\infty} E\left(z / a_{m}, p\right)$ converges; we make use of this in the theorems and proposition below.

Theorem 1. If $V \in C_{c}^{\infty}\left(\mathbb{R}^{n}\right)$ is real-valued, $V \not \equiv 0, n \geq 3$ odd, then as $r \rightarrow \infty$, $N(r)=\Omega\left(r(\log r)^{-p}\right)$ for any $p>1$.

Proof. Suppose $N(r)=\mathcal{O}\left(r(\log r)^{-p}\right)$ for some $p>1$. Then we may write

$$
s(\lambda)= \pm e^{i g(\lambda)} \prod \frac{\lambda_{j}+\lambda}{\lambda_{j}-\lambda},
$$

where this $g$ is perhaps different from the previous one, but has the same properties. 
Using the fact that if $\lambda_{j}$ is a resonance with non-zero real part, then $-\overline{\lambda_{j}}$ is also a resonance, we have for real $\lambda$

$$
\begin{aligned}
2 \pi \sigma(\lambda) & =g(\lambda)-\sum \int_{0}^{\lambda} \frac{2 \operatorname{Im} \lambda_{j}}{\left(\tau-\operatorname{Re} \lambda_{j}\right)^{2}+\left(\operatorname{Im} \lambda_{j}\right)^{2}} d \tau \\
& =g(\lambda)-2 \sum\left(\operatorname{Arctan}\left(\frac{\lambda-\operatorname{Re} \lambda_{j}}{\operatorname{Im} \lambda_{j}}\right)-\operatorname{Arctan}\left(\frac{-\operatorname{Re} \lambda_{j}}{\operatorname{Im} \lambda_{j}}\right)\right) .
\end{aligned}
$$

Again using the symmetry of the poles, we have

$$
\begin{aligned}
2 \pi \sigma(\lambda)=g(\lambda) & -2 \sum_{\operatorname{Re} \lambda_{j}=0} \operatorname{Arctan}\left(\frac{\lambda}{\operatorname{Im} \lambda_{j}}\right) \\
& -2 \sum_{\operatorname{Re} \lambda_{j}>0}\left(\operatorname{Arctan}\left(\frac{\lambda-\operatorname{Re} \lambda_{j}}{\operatorname{Im} \lambda_{j}}\right)+\operatorname{Arctan}\left(\frac{\lambda+\operatorname{Re} \lambda_{j}}{\operatorname{Im} \lambda_{j}}\right)\right) .
\end{aligned}
$$

This could also be obtained directly. The term involving the arctangent is the sum of the arguments of $\left(\lambda_{j}+\lambda\right)\left(\lambda_{j}-\lambda\right)^{-1}$ and $\left(-\overline{\lambda_{j}}+\lambda\right)\left(-\overline{\lambda_{j}}-\lambda\right)^{-1}$ if $\operatorname{Re} \lambda_{j}>0$, minus the value of the arguments when $\lambda=0$.

Using the fact that for $x>0$,

$$
\operatorname{Arctan} x+\operatorname{Arctan} y=\operatorname{Arctan} \frac{x+y}{1-x y}+ \begin{cases}0 & \text { if } x y<1 \\ \pi & \text { if } x y>1\end{cases}
$$

we obtain, when $|\lambda| \neq\left|\lambda_{j}\right|, \operatorname{Re} \lambda_{j}>0$,

(1) $\operatorname{Arctan}\left(\frac{\lambda-\operatorname{Re} \lambda_{j}}{\operatorname{Im} \lambda_{j}}\right)+\operatorname{Arctan}\left(\frac{\lambda+\operatorname{Re} \lambda_{j}}{\operatorname{Im} \lambda_{j}}\right)$

$$
=\operatorname{Arctan}\left(\frac{2 \lambda \operatorname{Im} \lambda_{j}}{\left|\lambda_{j}\right|^{2}-\lambda^{2}}\right)+ \begin{cases}0 & \text { if }\left|\lambda_{j}\right|^{2}>\lambda^{2} \\ \pi & \text { if }\left|\lambda_{j}\right|^{2}<\lambda^{2}\end{cases}
$$

We wish to bound

$$
\begin{aligned}
& h(\lambda)=2 \sum_{\operatorname{Re} \lambda_{j}=0} \operatorname{Arctan}\left(\frac{\lambda}{\operatorname{Im} \lambda_{j}}\right) \\
& \quad+2 \sum_{\operatorname{Re} \lambda_{j}>0}\left(\operatorname{Arctan}\left(\frac{\lambda-\operatorname{Re} \lambda_{j}}{\operatorname{Im} \lambda_{j}}\right)+\operatorname{Arctan}\left(\frac{\lambda+\operatorname{Re} \lambda_{j}}{\operatorname{Im} \lambda_{j}}\right)\right),
\end{aligned}
$$

from above and below. From our bounds, we will then derive a contradiction to the known asymptotics of the scattering phase. 
For the bound from above, note that

$$
\begin{aligned}
\sum_{\substack{\operatorname{Re} \lambda_{j}=0 \\
\left|\lambda_{j}\right|<2 \lambda}} \operatorname{Arctan}\left(\frac{\lambda}{\operatorname{Im} \lambda_{j}}\right) & \\
+\sum_{\substack{\operatorname{Re} \lambda_{j}>0 \\
\left|\lambda_{j}\right|<2 \lambda}}\left(\operatorname{Arctan}\left(\frac{\lambda-\operatorname{Re} \lambda_{j}}{\operatorname{Im} \lambda_{j}}\right)\right. & \left.+\operatorname{Arctan}\left(\frac{\lambda+\operatorname{Re} \lambda_{j}}{\operatorname{Im} \lambda_{j}}\right)\right) \\
& \leq \frac{\pi}{2} N(2 \lambda) \leq C\left(1+\lambda(\log \lambda)^{-p}\right) .
\end{aligned}
$$

Using the fact that $|\operatorname{Arctan} x| \leq|x|$, we can, for large $\lambda$, bound

$$
\begin{aligned}
2 \sum_{\substack{\operatorname{Re} \lambda_{j}=0 \\
\left|\lambda_{j}\right|>2 \lambda}} \operatorname{Arctan}\left(\frac{\lambda}{\operatorname{Im} \lambda_{j}}\right) & \leq 2 \sum_{\substack{\operatorname{Re} \lambda_{j}=0 \\
\left|\lambda_{j}\right|>2 \lambda}}\left|\frac{\lambda}{\operatorname{Im} \lambda_{j}}\right| \\
& \leq 2 \lambda \int_{2 \lambda}^{\infty} r^{-1} \frac{d}{d r} N(r) d r \\
& \leq C \lambda(\log \lambda)^{-p}+C \int_{2 \lambda}^{\infty} r^{-1}(\log r)^{-p} d r \\
& \leq C \lambda(\log \lambda)^{-\epsilon},
\end{aligned}
$$

for some $\epsilon>0$, where $C$ is a positive constant whose value may change from line to line. A similar bound can be made for the second sum in (2), showing that $h(\lambda)=\mathcal{O}\left(\lambda(\log \lambda)^{-\epsilon}\right)$.

For the lower bound, we use the fact that if $\left|\lambda_{j}\right|<\lambda / 2, \operatorname{Im} \lambda_{j}>0$, and $\operatorname{Re} \lambda_{j}=0$, then $\operatorname{Arctan}\left(\lambda / \operatorname{Im} \lambda_{j}\right)>\pi / 4$. Since

$$
\pi+\operatorname{Arctan} \frac{2 \lambda \operatorname{Im} \lambda_{j}}{\left|\lambda_{j}\right|^{2}-\lambda^{2}}>\frac{\pi}{2}
$$

using (1) and the fact that the contribution to $h$ of the poles with positive imaginary part is positive, we obtain

$$
\frac{\pi}{2} N\left(\frac{\lambda}{2}\right)-2 \pi n_{e} \leq h(\lambda)
$$

where $n_{e}$ is the sum of the number of eigenvalues and zero-resonances, counted with multiplicity. The non-zero eigenvalues correspond to the poles of the resolvent with negative imaginary part.

Summarizing, we have for large $\lambda$

$$
\frac{\pi}{2} N\left(\frac{\lambda}{2}\right)-2 \pi n_{e} \leq h(\lambda) \leq C \lambda(\log \lambda)^{-\epsilon} .
$$

Therefore, $h$ is unbounded but grows more slowly than linearly, and since $2 \pi \sigma(\lambda)=g(\lambda)-h(\lambda)$, this contradicts the known asymptotics of the scattering phase at infinity $([1,3,4])$. 
Set

$N_{I}(r)=\#\left\{\lambda_{j}\right.$ poles of $R(\lambda)$, counted with multiplicity : $\left.\left|\lambda_{j}\right|<r, \operatorname{Re} \lambda_{j}=0\right\}$.

The following result may be compared to [18, Example 5.3], which gives sufficient conditions for the Dirichlet or Neumann Laplacian on an exterior domain to have

$$
N(r)-N_{I}(r) \geq c_{\epsilon} r^{n-1-\epsilon},
$$

for any $\epsilon>0$.

Theorem 2. Let $V \in C_{c}^{\infty}\left(\mathbb{R}^{n}\right)$ be real-valued, $n \geq 3$ odd, and suppose $N_{I}(r) \geq$ $c r^{n-1}$ for some $c>0$. Then either $N(r)-N_{I}(r)=\Omega\left(r^{n-1}(\log r)^{-p}\right)$ for any $p>1$ or $N_{I}(r)=\Omega\left(r^{n}(\log r)^{-p}\right)$, any $p>1$.

Note that the results of $[9,19]$ show that the class of potentials satisfying the hypotheses of the theorem includes certain potentials of fixed sign.

Proof. Suppose that $N(r)=\mathcal{O}\left(r^{n}(\log r)^{-p}\right)$, some $p>1$. Then we may write $s(\lambda)= \pm e^{i g(\lambda)} P(-\lambda) / P(\lambda)$ with

$$
P(\lambda)=\prod_{\lambda_{j} \text { pole }, \lambda_{j} \neq 0} E\left(\lambda / \lambda_{j}, n-1\right) .
$$

Considering only the contribution of the purely imaginary poles to the scattering phase, we will show below that for large $\lambda$,

$$
\begin{aligned}
& \int_{1} \lambda^{n-1} \leq \\
& \int_{0}^{\lambda} \prod_{\substack{\lambda_{j} \text { pole } \\
\lambda_{j} \neq 0, \operatorname{Re} \lambda_{j}=0}} \frac{E\left(\tau / \lambda_{j}, n-1\right)}{E\left(-\tau / \lambda_{j}, n-1\right)} \frac{\partial}{\partial \tau}\left(\prod_{\substack{\lambda_{j} \text { pole } \\
\lambda_{j} \neq 0, \operatorname{Re} \lambda_{j}=0}} \frac{E\left(-\tau / \lambda_{j}, n-1\right)}{E\left(\tau / \lambda_{j}, n-1\right)}\right) d \tau \mid \\
& \quad=\left|\sum_{\substack{\lambda_{j} \text { ole }, \lambda_{j} \neq 0 \\
\operatorname{Re} \lambda_{j}=0}} \int_{0}^{\lambda} \frac{2 i \tau^{n-1}(-1)^{(n-3) / 2}}{\left(\operatorname{Im} \lambda_{j}\right)^{n-2}\left(\tau^{2}+\left(\operatorname{Im} \lambda_{j}\right)^{2}\right)} d \tau\right| \leq C_{2} \lambda^{n}(\log \lambda)^{-\epsilon},
\end{aligned}
$$

for some $C_{1}, C_{2}, \epsilon>0$. If we have $N(r)-N_{I}(r)=\mathcal{O}\left(r^{n-1}(\log r)^{-p}\right)$ for some $p>1$, then we have, when $\lambda$ is large,

$$
\begin{aligned}
& \left|\int_{0}^{\lambda} \prod_{\substack{\lambda_{j} \text { pole } \\
\operatorname{Re} \lambda_{j} \neq 0}} \frac{E\left(\tau / \lambda_{j}, n-1\right)}{E\left(-\tau / \lambda_{j}, n-1\right)} \frac{\partial}{\partial \tau}\left(\prod_{\substack{\lambda_{j} \text { pole } \\
\operatorname{Re} \lambda_{j} \neq 0}} \frac{E\left(-\tau / \lambda_{j}, n-1\right)}{E\left(\tau / \lambda_{j}, n-1\right)}\right) d \tau\right| \\
& \leq C \lambda^{n-1}(\log \lambda)^{-\epsilon},
\end{aligned}
$$

for some $C, \epsilon>0$. The proof of this estimate is very similar to that of (2) and (5), using, of course, the bound $\mathcal{O}\left(r^{n-1}(\log r)^{-p}\right)$ on the number of poles with nonzero real part. This estimate, combined with (5) and the fact that $g$ is 
an odd polynomial, contradicts the known asymptotics of the scattering phase $([1,3,4])$, which has highest order term $\lambda^{n-2}$. Therefore, at least one of our assumptions is incorrect.

We have now finished the proof, except for showing (5). To prove the lower bound, note that, using $N_{I}(r) \geq c r^{n-1}$,

$$
\begin{aligned}
\sum_{\substack{\lambda_{j} \text { pole, Re } \lambda_{j}=0 \\
\operatorname{Im} \lambda_{j}>0,\left|\lambda_{j}\right|<\lambda}} & \int_{0}^{\lambda} \frac{2 \tau^{n-1}}{\left(\operatorname{Im} \lambda_{j}\right)^{n-2}\left(\tau^{2}+\left(\operatorname{Im} \lambda_{j}\right)^{2}\right)} d \tau \\
& \geq \int_{0}^{\lambda} \int_{0}^{\lambda} \frac{2 \tau^{n-1}}{r^{n-2}\left(\tau^{2}+r^{2}\right)} d \tau \frac{d}{d r} N_{I}(r) d r-C \\
& \geq \int_{0}^{\lambda} \frac{2 c \lambda \tau^{n-1}}{\tau^{2}+\lambda^{2}} d \tau-\int_{0}^{\lambda} \int_{0}^{\lambda} \frac{d}{d r} \frac{2 \tau^{n-1}}{r^{n-2}\left(\tau^{2}+r^{2}\right)} d \tau N_{I}(r) d r-C \\
& \geq 2 c \lambda \int_{0}^{\lambda} \frac{\tau^{n-1}}{\tau^{2}+\lambda^{2}} d \tau-C \\
& \geq C^{\prime} \lambda^{n-1}-C
\end{aligned}
$$

where $C^{\prime}>0$, and here and below $C$ is a constant which may change from line to line.

For the upper bound in (5), note that much as in (4) and (6), using the upper bound on $N(r)$ it suffices to bound from above

$$
\int_{2}^{\infty} \int_{0}^{\lambda} \frac{\tau^{n-1}}{\tau^{2}+r^{2}} r(\log r)^{-p} d \tau d r
$$

If we write this as the sum of two integrals, where in the first we integrate over $r \in(2, \lambda)$ and the second $r \in(\lambda, \infty)$, we get for the first

$$
\begin{aligned}
\int_{2}^{\lambda} \int_{0}^{\lambda} \frac{\tau^{n-1}}{\tau^{2}+r^{2}} & r(\log r)^{-p} d \tau d r \\
& \leq \int_{2}^{\lambda} \int_{0}^{\lambda} \tau^{n-3} r(\log r)^{-p} d \tau d r \\
& \leq C \lambda^{n-2} \int_{2}^{\lambda} r(\log r)^{-p} d r \leq C \lambda^{n}(\log \lambda)^{-p}
\end{aligned}
$$


for large $\lambda$. For the part of the integral (7) with $\lambda \leq r<\infty$, we obtain, after a change of variables,

$$
\int_{1}^{\infty} \int_{0}^{1} \frac{\lambda^{n} \tau^{n-1} r}{r^{2}+\tau^{2}}(\log (r \lambda))^{-p} d \tau d r \leq C \lambda^{n}(\log \lambda)^{-\epsilon},
$$

for some $\epsilon>0$.

We include several results, for scattering by metric perturbations and obstacles, whose proofs are much the same. We continue to use $N(r)$ to denote the counting function for the poles, although we consider different kinds of perturbations of the Laplacian.

Proposition. If the resolvent of the Laplacian associated to a smooth, compactly supported, non-trapping metric perturbation of $\mathbb{R}^{n}, n \geq 3$ odd, has any poles, then $N(r)=\Omega\left(r(\log r)^{-p}\right)$, any $p>1$.

Proof. This proof follows just as the proof of Theorem 1, using the results of [11] for the asymptotics of the scattering phase. The proof works because of the existence of a complete asymptotic expansion of the scattering phase.

We note that $[15,17]$ have shown that if $n=3$ or $n=5$, then there are infinitely many resonances for a non-flat, compactly supported metric perturbation of $\mathbb{R}^{n}$. For general odd $n,[15]$ reduced the question of the existence of infinitely many resonances to the question of whether the Minakshisundaram coefficients $d_{j}, j \geq 2$, are 0 .

Examples. In [10], Levitin and Vassiliev constructed examples of bounded domains $\tilde{\Omega} \subset \mathbb{R}^{n}$ with interior Minkowski dimension $\tilde{D}, n-1<\tilde{D}<n$, such that

$$
N_{e v}(\mu)=c_{n} \operatorname{Vol} \tilde{\Omega} \mu^{n}+\tilde{c}_{\tilde{\Omega}, n} \mu^{\tilde{D}}+o\left(\mu^{\tilde{D}}\right)
$$

([10, Theorem C]), with

$$
\begin{aligned}
N_{e v}(\mu)=\#\left\{\lambda_{j}^{2} \leq \mu^{2}: \lambda_{j}^{2} \text { is an eigenvalue of the Laplacian on } \tilde{\Omega}\right. \\
\\
\text { with Dirichlet boundary conditions, counted with multiplicity }\},
\end{aligned}
$$

and $\tilde{c}_{\tilde{\Omega}, n} \neq 0$. (See [10] for a definition of interior and exterior Minkowski dimension.) Using their examples and a result of [2], it is relatively easy to construct families of obstacles $\Omega$ in $\mathbb{R}^{n}$ with exterior Minkowski dimension $\hat{D}$, $n-1<\hat{D}<n$, such that the Laplacian on $\mathbb{R}^{n} \backslash \Omega$ with Dirichlet boundary conditions has scattering phase with the asymptotics

$$
\sigma(\lambda)=c_{n} \lambda^{n} \operatorname{Vol}(\Omega) \lambda^{n}+\hat{c}_{\Omega, n} \lambda^{\hat{D}}+o\left(\lambda^{\hat{D}}\right),
$$

with $\hat{c}_{\Omega, n} \neq 0$ (see [2]). For such obstacles, when $n \geq 3$, odd, we have $N(r)=$ $\Omega\left(r^{\hat{D}}(\log r)^{-p}\right)$ for any $p>1$. Again, the proof is by contradiction. If $N(r)=$ 
$\mathcal{O}\left(r^{\hat{D}}(\log r)^{-p}\right)$ for some $p>1$, then we can write $s(\lambda)= \pm e^{i g(\lambda)} P(-\lambda) / P(\lambda)$, with

$$
P(\lambda)=\prod_{0 \neq \lambda_{j}, \text { pole }} E\left(\lambda / \lambda_{j}, n-1\right)
$$

Again using the bound $N(r)=\mathcal{O}\left(r^{\hat{D}}(\log r)^{-p}\right)$, we obtain, as $\lambda \rightarrow \infty, s(\lambda)=$ $\alpha_{n} \lambda^{n}+\mathcal{O}\left(\lambda^{\hat{D}}(\log \lambda)^{-\epsilon}\right)$ for some $\epsilon>0$, a contradiction.

Acknowledgments. It is a pleasure to thank R.B. Melrose, M. Zworski, and the referee for their comments on earlier versions of this note, and M. Zworski for helpful discussions.

\section{References}

[1] V.S. Buslaev, Trace formulas for Schrödinger's operator in three-space, Dokl. Akad. Nauk. SSSR 143 (1962), 1067-1070; Soviet Phys. Dokl. 7 (1962), 295-297.

[2] T. Christiansen, Spectral asymptotics for compactly supported perturbations of the Laplacian on $\mathbb{R}^{n}$, Comm. Partial Differential Equations 23 (1998), 933-948.

[3] Y. Colin de Verdière, Une formule de traces pour l'opérateur de Schrödinger dans $\mathbb{R}^{3}$, Ann. Sci. École Norm. Sup. (4) 14 (1981), 27-39.

[4] L. Guillopé, Asymptotique de la phase de diffusion pour l'operateur de Schrödinger avec potentiel, C.R. Acad. Sci. Paris Sér. I Math. 293 (1981), 601-603.

[5] L. Guillopé and M. Zworski, Scattering asymptotics for Riemann surfaces, Ann. of Math. (2) 145 (1997), 597-660.

[6] A. Jensen, Resonances in an abstract analytic scattering theory, Ann. Inst. H. Poincaré Sect. A (N.S.) 33 (1980), 209-223.

[7] A. Jensen, Spectral properties of Schrödinger operators and time-decay of the wave functions; results in $L^{2}\left(\mathbb{R}^{m}\right), m \geq 5$, Duke Math. J. 47 (1980), 57-80.

[8] A. Jensen and T. Kato, Spectral properties of Schrödinger operators and time-decay of the wave functions, Duke Math. J. 46 (1979), 583-612.

[9] P.D. Lax and R.S. Phillips, Decaying modes for the wave equation in the exterior of an obstacle, Comm. Pure Appl. Math. 22 (1969), 737-787.

[10] M. Levitin and D. Vassiliev, Spectral asymptotics, renewal theorem, and the Berry conjecture for a class of fractals, Proc. London Math. Soc. (3) 72 (1996), 188-214.

[11] A. Majda and J. Ralston, An analogue of Weyl's formula for unbounded domains III, an epilogue, Duke Math. J. 46 (1979), 725-731.

[12] R.B. Melrose, Geometric scattering theory, Stanford lectures, Cambridge University Press, Cambridge, 1995.

[13] W. Müller, Spectral geometry and scattering theory for certain complete surfaces of finite volume, Invent. Math. 109 (1992), 265-305.

[14] L.B. Parnovski, Spectral asymptotics of Laplace operators on surfaces with cusps, Math. Ann. 303 (1995), 281-296.

[15] A. Sá Barreto and S.-H. Tang, Existence of resonances in metric scattering, J. Comput. Appl. Math. 17 (1998), 3-18.

[16] A. Sá Barreto and M. Zworski, Existence of resonances in three dimensions, Comm. Math. Phys. 173 (1995), 401-415.

[17] A. Sá Barreto and M. Zworski, Existence of resonances in potential scattering, Comm. Pure Appl. Math. 49 (1996), 1271-1280.

[18] J. Sjöstrand and M. Zworski, Lower bounds on the number of scattering poles, II, J. Funct. Anal. 123 (1994), 336-367.

[19] A. Vasy, Scattering poles for negative potentials, Comm. Partial Differential Equations 21 (1997), 185-194. 
[20] M. Zworski, Distribution of poles for scattering on the real line, J. Funct. Anal. 73 (1987), $277-296$.

[21] M. Zworski, Sharp polynomial bounds on the number of scattering poles, Duke Math. J. 59 (1989), 311-323.

[22] M. Zworski, Poisson formulae for resonances, Séminaire sur les Équations aux Dérivées Partielles, 1996-1997, Exp. No. XIII, École Polytech., Palaiseau, 1997.

[23] M. Zworski, Poisson formula for resonances in even dimensions, Asian J. Math. 2 (1998), $615-624$

Department of Mathematics, University of Missouri, Columbia, Missouri 65211

E-mail address: tjc@math.missouri.edu 